\title{
Development and Evaluation of Droplet Digital PCR Assay for the Detection of Reticuloendotheliosis Virus in Attenuated Vaccines
}

Fanfeng Meng

China Agricultural University

\section{Zhihao Ren}

China Agricultural University

\section{Yixin Wang}

Shandong Agricultural University

Peng Zhao

Shandong Agricultural University

Guozhong Zhang ( $\nabla$ zhanggz@cau.edu.cn )

China Agricultural University

\section{Research Article}

Keywords: Reticuloendotheliosis virus (REV), exogenous virus, attenuated vaccine, droplet digital PCR

Posted Date: August 9th, 2021

DOl: https://doi.org/10.21203/rs.3.rs-763067/v1

License: (9) This work is licensed under a Creative Commons Attribution 4.0 International License. Read Full License 


\section{Abstract}

Background: The use of Reticuloendotheliosis virus (REV) from contaminated live virus vaccine is suspected to be one of the most important causes of massive outbreaks of Reticuloendotheliosis in China.

Methods: In this study, we established a droplet digital PCR (ddPCR) detection method for REV and compared its sensitivity to different methods to detect REV contamination in a vaccine.

Results: The results indicated that both quantitative PCR and dot-blot methods could detect REV contamination at a dose of $1 \mathrm{TCID}_{50} / 1,000$ feathers, whereas ddPCR could detect REV contamination at a dose of $0.1 \mathrm{TCID}_{50} / 1,000$ feathers, which is 1,000 -fold more sensitive than conventional polymerase chain reaction detection ( $10^{2} \mathrm{TCID}_{50} / 1000$ feathers). ddPCR not only exhibited the highest sensitivity but also proved extremely intuitive, especially to detect REV contamination in vaccines.

Conclusions: The ddPCR method established in this study to detect REV contamination in vaccines can effectively detect and quantify low-dose REV contamination. This provides a new method for the rapid detection of REV contamination in various samples, especially vaccines.

\section{Introduction}

Reticuloendotheliosis (RE), causing neoplastic and immunosuppressive disease by avian Reticuloendotheliosis virus (REV), has caused immense economic losses all over the world in the poultry industry [1, 2]. The host of REV included chickens, turkeys, ducks and geese. REV mainly infected and transmitted chickens horizontally and vertically [3]. Moreover, the misuse of REV-contaminated attenuated virus vaccines was considered the most likely cause of the widespread of $\operatorname{REV}[4,5,6,7]$.

In the last 20 years, many attenuated vaccines for poultry have been confirmed to contain exogenous viruses. This is mainly because of the use of vaccines synthesized from SPF chicken embryos contaminated with viruses, for example, $\operatorname{ALV}[8,9,10,11], \operatorname{CIAV}[1]$, and $\operatorname{REV}[2,12,13,14,15,16,17]$, which are mainly vertically transmitted. One of the major differences between the detection of exogenous viruses in vaccines and in clinical samples is that the main component of the vaccine is the vaccine strain. In general, the dose of the exogenous virus only occupies a relatively small proportion of the total nucleic acid which is likely to cause misdetection; therefore, a high-sensitivity detection method is urgently required. Droplet digital PCR (ddPCR) is a newly developed implementation of digital PCR that facilitates the accurate and precise quantitation of nucleic acid targets without the need for calibration curves $[6,18]$. The sensitivity of ddPCR is much higher than that of real-time quantitative PCR and other methods. This study concluded that ddPCR is an effective diagnostic technology suitable for both research and clinical use in detecting REV, particularly in attenuated live vaccines.

\section{Materials And Methods}




\subsection{Virus strains and vaccines}

The REV strain IBD-C1605 (GenBank accession number KX278301) was isolated from the virus seed of a contaminated attenuated infectious bursal disease virus (IBDV) vaccine in China in 2016 [2]. This strain exhibited a median tissue culture infective dose $\left(\mathrm{TCID}_{50}\right)$ of $10^{3.5}$ per $0.1 \mathrm{~mL}$ of chicken embryo fibroblast cell culture supernatant. Marek's disease virus (MDV), IBDV, Newcastle disease virus (NDV), fowlpox virus (FPV), and infectious bronchitis virus (IBV) vaccines were purchased commercially and were all qualified as attenuated vaccines lacking exogenous viruses by the China Institute of Veterinary Drug Control and used as negative controls.

\subsection{Primer and probe design}

Primers P1/P2, which targeted the pol gene of REV, were based on the published sequences of REV and designed using Primer 5.0 for amplification by polymerase chain reaction (PCR). Both real-time PCR and ddPCR used published primers and probes to conserved regions of the REV pol gene [19]. All the primers and probes were synthesized by the Shanghai Bioengineering Company (Shanghai, China) and are listed in supplementary Table 1.

\subsection{Plasmid preparation}

The genomic REV DNA from cells inoculated with the IBD-C1605 isolate were used as a template. PCR was performed in a $50-\mu \mathrm{L}$ reaction volume, and conditions were as follows: initial denaturation for 5 min at $95{ }^{\circ} \mathrm{C}, 31$ cycles of denaturation for $30 \mathrm{~s}$ at $95^{\circ} \mathrm{C}$, annealing for $30 \mathrm{~s}$ at $58{ }^{\circ} \mathrm{C}$, and an extension for 30 $\mathrm{s}$ at $72{ }^{\circ} \mathrm{C}$ followed by a final extension for $10 \mathrm{~min}$ at $72{ }^{\circ} \mathrm{C}$. The amplified products were separated by $1 \%$ agarose gel electrophoresis and purified using an E.Z.N.A Gel Extraction kit (OMEGA, USA). The purified PCR products were cloned into a pMD18-T vector (Takara, Japan) and transformed into competent Escherichia coli DH5a cells. All single clone plasmids were extracted using a Plasmid Mini Kit (Omega Biotek) and identified by restriction analysis. Positive clones were sequenced by the Shanghai Bioengineering Company (Shanghai, China). The positive recombinant plasmid verified by sequencing was used as a standard and named PMD18-T-pol. The concentration of the plasmid was measured using a DNA quantitative instrument and used to calculate the copy number. The recombinant plasmid was diluted in a tenfold serial gradient and stored at $-20{ }^{\circ} \mathrm{C}$.

\subsection{Optimization of ddPCR assay}

Droplet digital PCR reaction consists of four steps: preparation of the reaction system, droplet generation, droplet cycling (PCR), and droplet reading [20]. However, exploration of different primer and probe concentrations and annealing temperatures is needed to identify optimal reaction conditions. ddPCR reaction mixtures were prepared as follows: $10 \mu \mathrm{L}$ of $2 \times$ ddPCR master mix, $1.8 \mu \mathrm{L}$ of relevant forward and reverse primers, $0.8 \mu \mathrm{L}$ of probe, $2 \mu \mathrm{L}$ of template, and $3.6 \mu \mathrm{L}$ of nuclease- and protease-free water were mixed to produce a $20-\mu \mathrm{L}$ reaction volume. The conditions were $3 \mathrm{~min}$ at $95^{\circ} \mathrm{C}$, followed by 40 cycles of $95{ }^{\circ} \mathrm{C}$ for $10 \mathrm{~s}, 58^{\circ} \mathrm{C}$ for $40 \mathrm{~s}$, and $98^{\circ} \mathrm{C}$ for $10 \mathrm{~min}$. The ramp rate was fixed to $2.5^{\circ} \mathrm{C} / \mathrm{s}$. 
According to the generation number, distribution, and fluorescence signal of the droplet, the assays need to identify the optimal results.

\subsection{Specificity, sensitivity, and reproducibility of ddPCR assays}

The specificity of the established ddPCR method was evaluated using different reference virus strains for DNA or cDNA as templates, including REV-HA9901 (GenBank accession No. AY842951), ALV-ASDAU09C1 (GenBank accession No. HM452339), ALV-J-NX0101 (GenBank accession No. DQ115805), CIAV-SDLY08 (GenBank accession No. FJ172347), MDV-GX0101 (GenBank accession No. JX844666), and FAdV-4 N-22 (GenBank accession No. KU764777). The assay was also evaluated using DNA or cDNA templates from attenuated vaccines, such as those for MDV, IBDV, NDV, FPV, and IBV. All plasmid standards were used to produce tenfold dilution gradients and tested by PCR, dot-blot hybridization [21], real-time quantitative PCR (qPCR) [19], and ddPCR to compare the sensitivity of the different assays. The intra- and inter-batch reproducibility and stability of the ddPCR assay were also analyzed using three independent ddPCR assays for each of the above samples under identical conditions.

\subsection{Detection of REV contamination in attenuated vaccines with different doses of REV using different methods}

Quantified IBD-C1605 was diluted to 1,000, 100, 10, 1, 0.1, and $0.01 \mathrm{TCID}_{50} / \mathrm{mL}$ with sterile phosphatebuffered saline (PBS). A total of 6 bottles of IBDV vaccine (1,000 feathers) from the same batch were diluted using the same PBS solution containing HA9901 at different titers. The samples were then centrifuged at 2,000 rpm for 2 min at $4{ }^{\circ} \mathrm{C}$, and $200 \mu \mathrm{L}$ of each virus-containing supernatant was used to extract total RNA using a Viral RNA Kit (OMEGA, USA). cDNAs were generated from the viral RNAs by reverse transcription and PCR. All cDNAs from the six vaccine bottles vaccines were tested by PCR, dotblot hybridization, qPCR, and ddPCR for comparison of sensitivity between the different assays.

\section{Results}

\subsection{Development of qPCR for REV}

PCR analysis showed that PMD18-T-pol contained a 1500 bp fragment, which was consistent with its expected size. Thus, target fragment had been correctly inserted into the vector. DNA sequencing further confirmed its identity, with the homology of the amplified fragment to the reference sequence being $100 \%$. The plasmid standard was extracted, and it was DNA determined to be $350 \mu \mathrm{g} / \mathrm{mL}$. This positive control recombinant plasmid was diluted in a tenfold gradient from $10^{7}$ copies to $10^{1}$ copies per $\mu \mathrm{L}$.

The ddPCR assay for REV was optimized at various annealing temperatures and exhibited slightly better efficiency at $60^{\circ} \mathrm{C}$ compared to lower temperatures (data not shown). This ddPCR exhibited high specificity for the REV DNA and did not cross-react with other tested viral DNA, namely, ALV, CIAV, and FADV. Each standard plasmid was tenfold serially diluted four times, and the reaction efficiency at each 
dilution was calculated. Thus, ddPCR could accurately detect the plasmid standard down to as low as one copy, indicating good sensitivity (Fig. 1). In addition, ddPCR also exhibited good specificity, since no amplification signals were detected using DNA or cDNA from other viruses.

\subsection{Detection of REV contamination using different methods in attenuated IBDV vaccines}

Conventional PCR tests of vaccines contaminated with REV at different doses produced positive results only at $100 \mathrm{TCID}_{50}$ (Fig. 2A). Dot-blots could consistently detect contamination at a dose of $1 \mathrm{TCID}_{50}$ for each vaccine (Fig. 2B), while qPCR could consistently detect more than $1 \mathrm{TCID}_{50}$ of REV contamination (Fig. 2C), but the $\mathrm{Ct}$ value of $0.1 \mathrm{TCID}_{50}$ REV per vaccine and blank control greater than 37 can be considered as negative, and the nucleic acid detection of different vaccines revealed no positive fluorescence signal.

Clinical samples contaminated with different doses of REV and that produced specific qPCR signals were used for the evaluation of the efficiency of the ddPCR assay based on standard curve analysis. Each cDNA sample underwent five serial 10-fold dilutions, and least 10,000 droplets were generated from each sample (Fig. 3A). Few positive droplets were detected (less than one copy) from the blank vaccines (Fig. 3B), and only a single peak was observed after PCR (Fig. 3C). However, when REV contamination was above $0.1 \mathrm{TCID}_{50}$, the number of positive droplets detected was significantly higher than with the blank vaccine ( $p<0.05$; Fig. 3D), and so the positive sample exhibited two peaks (Fig. 3E). The amount of contamination was linear with the copy number (Fig. 3F), but the confidence level was unacceptable when vaccine contamination was below $0.01 \mathrm{TCID}_{50}$ per 1,000 feathers. The highest sensitivity of ddPCR was determined to be $0.1 \mathrm{TCID}_{50}$ per 1,000 feathers, which was 1,000 times higher than that of conventional PCR and >10 times that of both dot-blotting and qPCR (Table 1).

Table 1

comparison of the sensitivity for detecting REV with different detection methods

\begin{tabular}{|c|c|c|c|c|c|c|c|c|c|c|c|}
\hline \multirow[t]{2}{*}{ Detection } & \multicolumn{6}{|c|}{$\begin{array}{l}\text { Vaccines with different concentrations of } \\
\left.\text { REV (TCID }{ }_{50}\right)\end{array}$} & \multirow[t]{2}{*}{ NDV } & \multirow[t]{2}{*}{ FPV } & \multirow[t]{2}{*}{ MDV } & \multirow[t]{2}{*}{ IBDV } & \multirow[t]{2}{*}{$\mathrm{H}_{2} \mathrm{O}$} \\
\hline & $10^{3}$ & $10^{2}$ & $10^{1}$ & $10^{0}$ & $10^{-1}$ & $10^{-2}$ & & & & & \\
\hline $\begin{array}{l}\text { Conventional } \\
\text { PCR }\end{array}$ & + & + & - & - & - & - & - & - & - & - & - \\
\hline Dot-blot & + & + & + & + & - & - & - & - & - & - & - \\
\hline $\begin{array}{l}\text { Real-time } \\
\text { qPCR }\end{array}$ & + & + & + & + & - & - & - & - & - & - & - \\
\hline ddPCR & + & + & + & + & + & - & - & - & - & - & - \\
\hline
\end{tabular}

\section{Discussion}


A high positive frequency of REV antibody has been found in many Chinese chicken breeds, especially in certain local strains $[22,23,24]$ in recent years, indicating a serious risk of REV infection in China. Many REV outbreaks have been suspected to be due using attenuated REV-contaminated vaccines, be it in China or in other countries, and the pain culprits are thought to be fowlpox and Marek's disease vaccines $[9,12,14,17,25]$. Presently, REV contamination in vaccines is mainly detected using cell culture and SPF chicken inspection methods; however, this is difficult in fowlpox and Marek's vaccines owing to the cellular lesions of the virus vaccine, and the replication and isolation of REV being disturbed. Thus, REV contamination can be detected by the SPF chicken inspection method; however, this is costly, and its cycle is longer. Therefore, there has been great interest in detecting REV contamination in vaccines using molecular biological detection methods.

The primary components of the REV genome are gag, pol, env, and long terminal repeats (LTRs), and many Chinese researchers have established a series of quantitative fluorescence detection methods for different REV regions, such as gag, env, and LTR $[26,27,28]$. However, when vaccines were tested, many positive and vaccines were found to be virus-free or otherwise negated and only some integrated genetic were detected successfully rather than free virus of REV. It has long been acknowledged that the genetic components of REV can be integrated into the genomes of other viruses. The most reported of these are REV integration into the MDV viral genome $[5,29]$ and the natural recombinant wild-type strain of MDV (GX0101) that has been isolated in China containing an LTR gene fragment of REV. There has also been research regarding the integration of REV env gene and LTR fragments into FPV in China [30]. However, there have been no reports regarding the recombination of pol genes, which are very conserved in REV. Therefore, we established a real-time quantitative PCR method for detecting REV pol genes and applied it to the detection of REV contamination in attenuated vaccines.

In general, levels of REV contamination in avian attenuated vaccines are low which therefore place higher demands on the sensitivity and stability of assays to detect them. Furthermore, it should be simple and intuitive for the analyst to judge the difference between contaminated and uncontaminated vaccines. The establishment and application of qPCR assays require the use of a standard curve, and its quantification is based on relative CT values rather than absolute quantification. In the actual tests, we often find that when qPCR test different blank vaccine samples, the qPCR CT value of the most attenuated vaccine is 37 or more, but some of their CT value are of precisely between 34 and 36 , and we cannot determine whether this because of the analyst's slight error or very low contamination of the vaccine. Furthermore, the repeatability of qPCR assays is poor at lower contaminant levels (Fig. 2B), making it difficult to assess CT-based results.

ddPCR is a new technology for the absolute quantification of nucleic acid molecules. Unlike qPCR, ddPCR does not rely on standard curves and standards and can directly determine nucleic acid copy number in a sample. Considering the generally low levels of exogenous virus contamination, we hypothesized that the ddPCR technique would exhibit greater speed and sensitivity than conventional PCR, dot-blotting and qPCR in detecting low-level contaminants in attenuated vaccines. We subsequently found that when 1,000 feathers of vaccine were contaminated with $0.1 \mathrm{TCID}_{50} \mathrm{REV}$, all the conventional methods failed to 
detect REV contamination consistently. However, REV contamination at $0.1 \mathrm{TCID}_{50} / 1,000$ feathers was detected by ddPCR, with very significant differences being observed even between contaminated vaccines with low REV copy numbers and uncontaminated vaccines (Fig. 4E). These differences were extremely obvious, suggesting that they could be used to make highly intuitive judgments with respect to contamination.

\section{Conclusions}

The ddPCR established in this study is highly sensitive and specific; thus it can rapidly detect very low levels of REV contamination in vaccines. The tests also allow for more intuitive judgment of positive results by an analysist than conventional PCR, qPCR, and dot-blotting. Thus, ddPCR is a viable method for exogenous virus detection and quantification which can more effectively promote the safe use of vaccines.

\section{Abbreviations}

Reticuloendotheliosis virus (REV); droplet digital PCR (ddPCR); Reticuloendotheliosis (RE); infectious bursal disease virus (IBDV); tissue culture infective dose (TCID50); Marek's disease virus (MDV); IBDV, Newcastle disease virus (NDV); fowlpox virus (FPV); infectious bronchitis virus (IBV); polymerase chain reaction (PCR); phosphate-buffered saline (PBS); long terminal repeats (LTRs); real-time quantitative PCR (qPCR).

\section{Declarations}

\section{Acknowledgments}

The authors would like to thank the laboratory teams of avian tumorigenic disease at Shandong agricultural university.

\section{Ethics approval and consent to participate}

Not applicable.

\section{Consent for publication}

Not applicable.

\section{Funding}

This work was supported by the National Key Research and Development Program of China (2016YFD0501606).

\section{Availability of data and materials}


The datasets used and/or analyzed during the current study are available from the corresponding author on reasonable request.

\section{Authors' contributions}

MFF and RZH designed and conducted the study, performed most of the experiments, and wrote the manuscript. WYX performed the calculation. ZP and ZGZ designed the study and wrote the manuscript. All authors read and approved the final manuscript.

\section{Conflict of Interest}

The authors declare that the research was conducted in the absence of any commercial or financial relationships that could be construed as a potential conflict of interest.

\section{References}

1. Li Y, Hu Y, Cui S, Fu JY, Wang YX, Cui ZZ, et al. Molecular characterization of chicken infectious anemia virus from contaminated live-virus vaccines. Poul. Sci. 2017; 96(5):1045-1051.

2. Li Y, Cui S, Cui Z, Chang S, Zhao P. Genome analysis and pathogenicity of reticuloendotheliosis virus isolated from a contaminated vaccine seed against infectious bursal disease virus, first report in China. J. Gen. Virol. 2016;97(11): 2809-2815.

3. Ren ZH, Meng FF, Li QC, Wang YX, Liu XF, Cui ZZ, et al. Protection induced by a gp90 protein-based vaccine derived from a Reticuloendotheliosis virus strain isolated from a contaminated IBD vaccine. Virology Journal. 2018;15(1):42.

4. Biswas SK, C Jana, K Chand, W Rehman, B Mondal. Detection of fowl poxvirus integrated with reticuloendotheliosis virus sequences from an outbreak in backyard chickens in India. Vet Ital. 2011;47(2):147-153.

5. Jones D, P Brunovskis, R Witter, HJ Kung. Retroviral insertional activation in a herpesvirus: transcriptional activation of US genes by an integrated long terminal repeat in a Marek's disease virus clone. J. Virol. 1996);70:2460-2467.

6. Pinheiro LB, Coleman VA, Hindson CM, Herrmann J, Hindson BJ, Bhat S. Evaluation of a droplet digital polymerase chain reaction format for DNA copy number quantification. Analytical Chemistry. 2012;84:1003-11.

7. Sun AJ, XY Xu, L Petherbridge, YG Zhao, V Nair, et al. Functional evaluation of the role of reticuloendotheliosis virus long terminal repeat (LTR) integrated into the genome of a field strain of Marek's disease virus. Virology. 2010;397(2): 270-276】

8. Barbosa T, Zavala G, Cheng S. Molecualr characterization of three recombinant isolates of avian leukosis virus obtained from contaminated Marek's disease vaccines. Avian Dis. 2008;52(2):245-252.

9. Fadly A, Silva R, H Hunt, A Pandiri, C Davis. Isolation and characterization of an adventitious avian leukosis virus isolated from commercial Marek's disease vaccines. Avian Dis. 2006;50(3):380-385. 
10. Zavala G, Cheng S. Detection and characterization of avian leukosis virus in Marek's disease vaccines. Avian Dis. 2006;50(2):209-215.

11. Zhao $P$, Dong $X$, Cui ZZ. Isolation, identification, and gp85 characterization of a subgroup $A$ avian leukosis virus from a contaminated live Newcastle Disease virus vaccine, first report in China. Poul. Sci. 2014;93(9):2168-2174.

12. Awad AM, Abd El-Hamid HS, Abou Rawash AA, Ibrahim HH. Detection of reticuloendotheliosis virus as a contaminant of fowl pox vaccines. Poul Sci. 2010; 89(11):2389-2395.

13. Fadly A, Garcia MC. Detection of reticuloendotheliosis virus in live virus vaccines of poultry. Dev Biol. 2006;126:301-305.

14. Li J, Dong X, Yang C, Li QH, Cui ZZ, Chang S, et al. Isolation, identification, and whole genome sequencing of reticuloendotheliosis virus from a vaccine against Marek's disease. Poul. Sci. 2015;94(4):643-649.

15. Liu QF, Zhao JX, Su JL, Pu J, Zhang GZ, Liu JH. Full genome sequences of two reticuloendotheliosis viruses contaminating commercial vaccines. Avian Dis. 2009;53(3):341-346.

16. Wang JY, Li ZM, Zhao P, Chen H, Cui ZZ. Isolation of Reticuloendotheliosis Virus from a fowlpox live vaccine and env gene sequence analysis. Chinese Journal of Animal Infectious Diseases. 2010;18:35-39.

17. Wei K, Sun Z, Zhu S, Guo WL, Sheng PC, et al., Probable congenital transmission of reticuloendotheliosis virus caused by vaccination with contaminated vaccines. PloS one. 2012;7(8):e43422.

18. Hindson, BJ, Ness KD, Masquelier DA, Belgrader P, Heredia NJ, Makarewicz AJ, et al. High-throughput droplet digital PCR system for absolute quantitation of DNA copy number. Anal. Chem. 2011;83(22):8604-8610.

19. Luan H, Wang Y, Li Y, Cui Z, Chang S, Zhao P. Development of a real-time quantitative RT-PCR to detect REV contamination in live vaccine. Poul. Sci. 2016;95(9):2023-2029.

20. Morisset D, Stebih D, Milavec M, Gruden K, Zel J. Quantitative analysis of food and feed samples with droplet digital pcr. Plos One. 2013;8(5):e62583.

21. Cui ZZ, Zhao P, Sun SH, Wang X, Li WP. Cross dot blot hybridization with specific nucleic acid probes for detection of pathogenic exogenous avian leucosis virus. Chinese Journal of Veterinary Drug. 2011;45(08):5-11.

22. Cheng Z, Y Shi, L Zhang, G Zhu, X Diao, ZZ Cui. Occurrence of reticuloendotheliosis in Chinese partridge. J Vet Med Sci. 2007;69:1295-1298.

23. Cui Z, S Sun, Z Zhang, S Meng. Simultaneous endemic infections with subgroup J avian leukosis virus and reticuloendotheliosis virus in commercial and local breeds of chickens. Avian Pathol. 2009;38(6):443-448.

24. Zhao P, C Ma, Y Du, ZZ Cui. Serological survey of the Reticuloendotheliosis virus infection in China native chicken flocks. Pak. Vet. J. 2012;32:621-623. 
25. Bagust TJ, TM Grimes, DP Dennett. Infection studies on a reticuloendotheliosis virus contaminant of a commercial Marek's disease vaccine. Aust Vet J. 1979; 55:153-157.

26. Li K, Gao HL, Gao L, Qi XL, Gao YL, et al. Development and preliminary application of a TaqMan realtime PCR assay for detection of reticuloendotheliosis virus. Chinese Veterinary Science. 2010;40(11): 1137-1141.

27. Miao J, Bao YQ, Shao HX, Qian K, Qin AJ. Development and preliminary application of a SYBR GREEN I based multiple real-time PCR assay for detection of reticuloendotheliosis virus in chicken embryo fibrolasts. Chinese Journal of Animal Infectious Diseases. 2015;23(1):1-8.

28. Zhao LQ, SF Wang, YG Wu, ZL Wang. Research of detection method of REV using real-time PCR. Chin. J. Vet. Sci. 2007;27:795-798.

29. Davidson I, R Borenshtain. In vivo events of retroiral long terminal repeat integration into Marek's disease virus in commerial poultry: detection of chimeric molecules as a marker. Avian Dis. 2001;45(1):102-121.

30. Ding JB, Cui ZZ, Yu LJ, Sun SH, Jiang SJ. Detection of Reticuloendotheliosis Virus Genomic Sequence in The Genome of Field and Vaccine Strains of Fowl Poxvirus. Acta Microbiologica Sinica Vol. 2004;05: 588-592.

\section{Figures}


A

Ch1 Pos:24140 Accepted: 171670

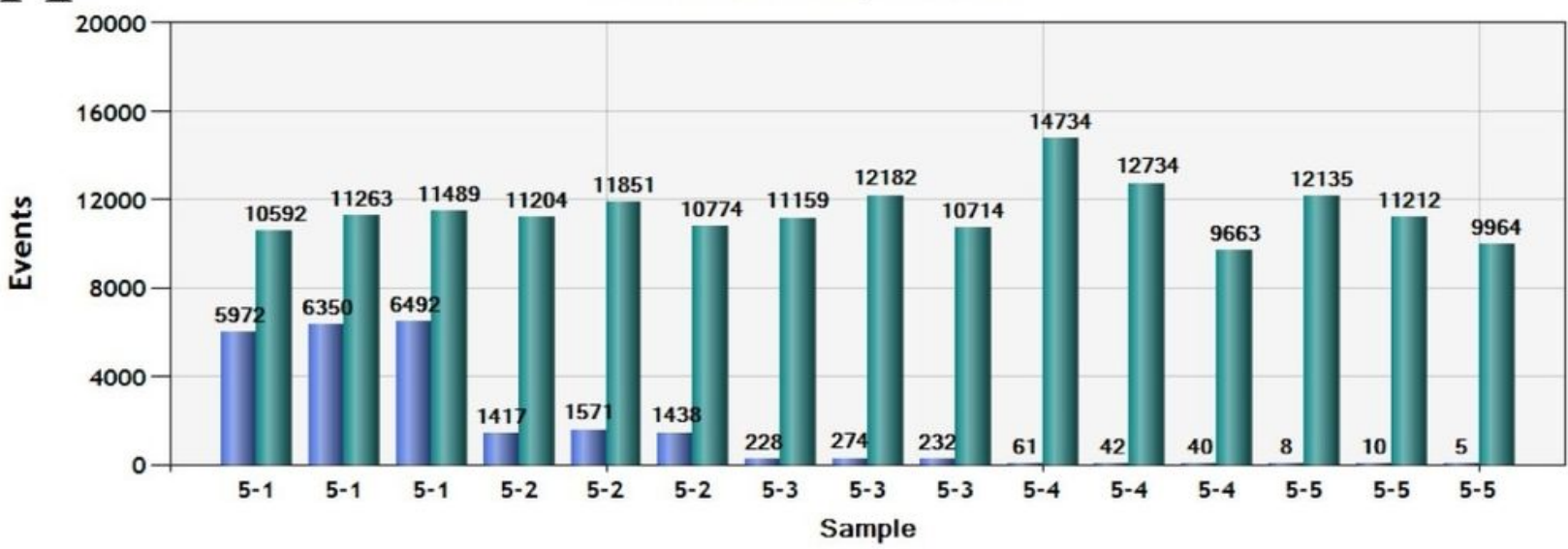

B
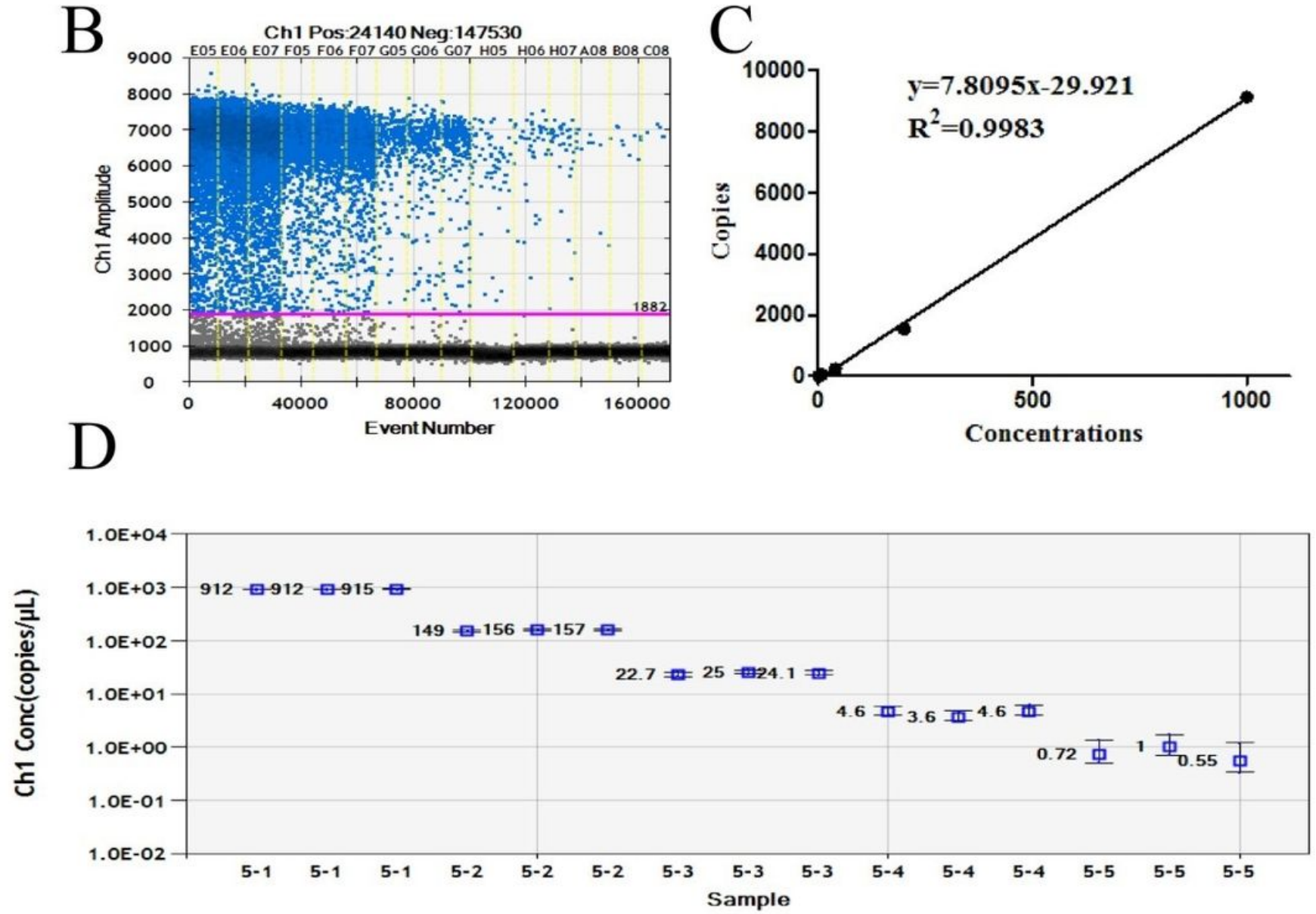

Figure 1

ddPCR for the standard plasmid (A) Positive and negative droplets. Low light blue is the number of positive droplets. High dark green is the number of negative droplets. (B) Results of droplet amplification with different concentrations of the standard samples. The blue above is the positive droplet and the black below is the negative droplet. (C) The standard curve of the ddPCR with different concentrations. (D) The copy number of samples with different concentrations. 

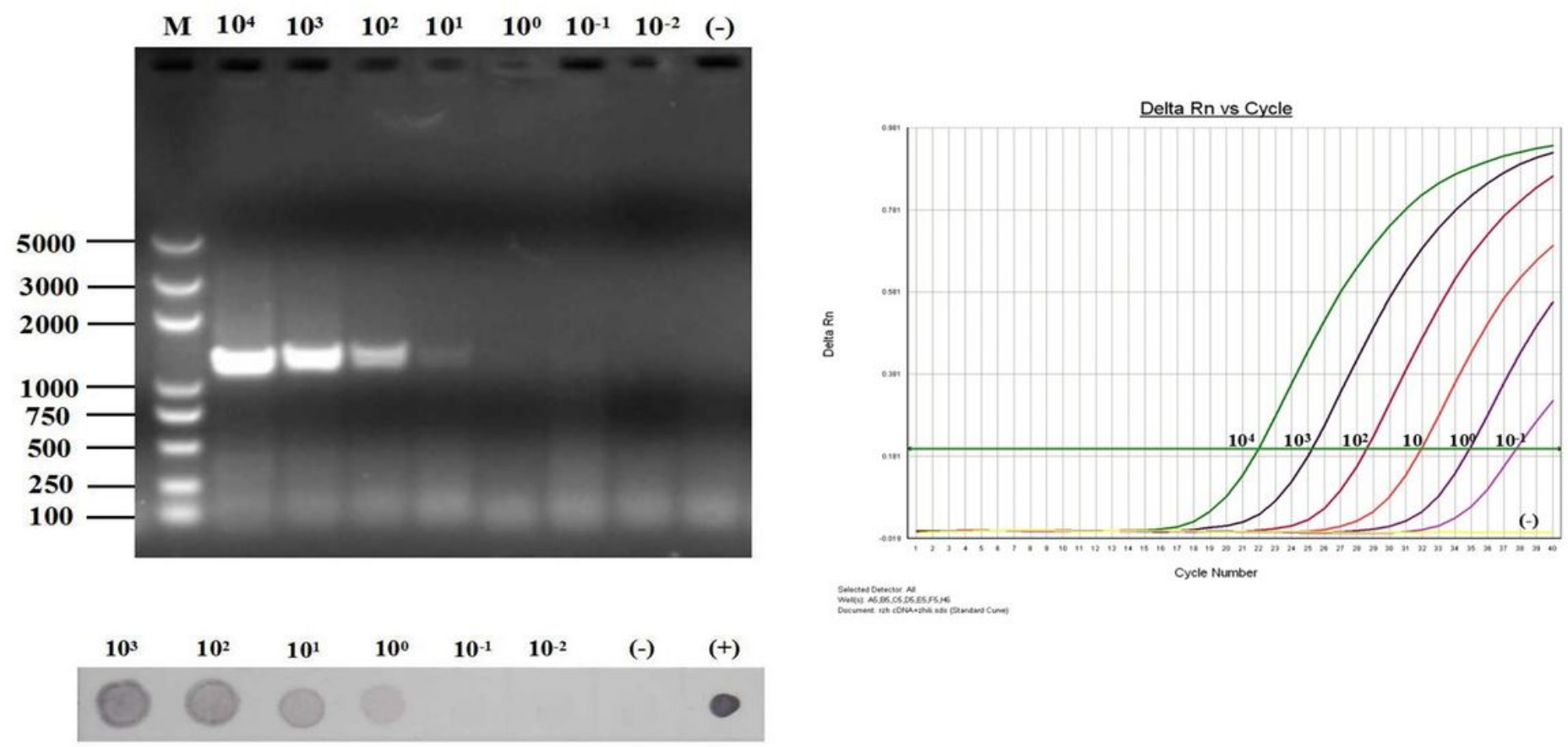

\section{Figure 2}

The sensitivity of routine PCR, dot-blot and qPCR in detecting REV in the contaminated vaccine (A) Marker, DL5000; 104-10-2 means REV contamination at a dose of 104-10-2 TCID50/1,000 feathers; (-) negative control. 

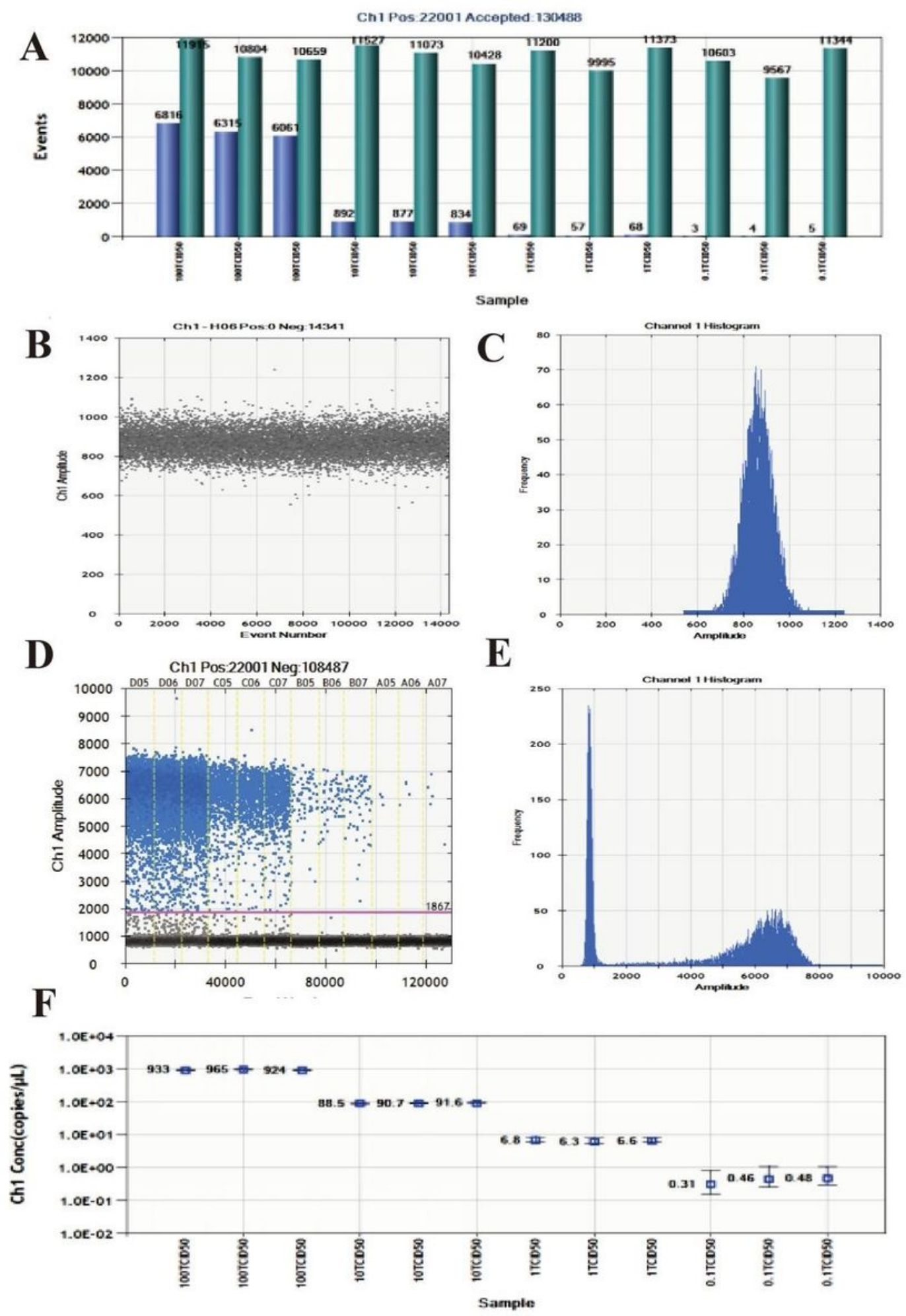

\section{Figure 3}

The construction and application of ddPCR for REV (A) Positive and negative droplets. Low light blue is the number of positive droplets. High dark green is the number of negative droplets. (B) Results of droplet amplification with blank samples. The black is the negative droplet. (C) Frequency distribution histogram, the cluster with low fluorescent intensity corresponds to the negative droplet. (D) Results of droplet amplification with different concentrations of the standard samples. The blue above is the positive 
droplet and the black below is the negative droplet. (E) Frequency distribution histogram, the left cluster with lower fluorescent intensity corresponds to the negative droplet, and the right side of the cluster with higher fluorescent intensity corresponds to the positive droplet. (F) The copy number of samples with different concentrations

\section{Supplementary Files}

This is a list of supplementary files associated with this preprint. Click to download.

- Supplementarytable.doc 